\title{
Robot-Assisted Prostate Brachytherapy
}

\author{
Yan $\mathrm{Yu}^{1,5}$, Tarun Podder ${ }^{1}$, Yongde Zhang ${ }^{1}$, Wan-Sing $\mathrm{Ng}^{6}$, Vladimir Misic ${ }^{1}$, \\ Jason Sherman ${ }^{1}$, Luke Fu ${ }^{1}$, Dave Fuller ${ }^{1}$, Edward Messing, ${ }^{2,4}$, Deborah Rubens ${ }^{3,4,5}$, \\ John Strang ${ }^{3,4}$, and Ralph Brasacchio ${ }^{1}$
}

\author{
Departments of \\ ${ }^{1}$ Radiation Oncology \\ ${ }^{2}$ Urology \\ ${ }^{3}$ Radiology and \\ ${ }^{4}$ Surgery, \\ University of Rochester, Rochester, NY 14642, USA \\ ${ }^{5}$ Department of Biomedical Engineering, \\ University of Rochester, Rochester, NY 14642, USA \\ ${ }^{6}$ School of Mechanical and Aerospace Engineering, \\ Nanyang Technological University, Singapore 639798
}

\begin{abstract}
In contemporary brachytherapy procedures, needle placement at the desired target is challenging due to a variety of reasons. A robot-assisted brachytherapy system can improve the needle placement and seed delivery resulting in enhanced patient care. In this paper we present a 16 DOF (degreesof-freedom) robotic system (9DOF positioning module and 7DOF surgery module) developed and fabricated for prostate brachytherapy. Techniques to reduce needle deflection and target movement have been incorporated after verifying with extensive experiments. Provisions for needle motion and force feedback have been included into the system for improving the robot control and seed delivery. Preliminary experimental results reveal that the prototype system is quite accurate (sub-millimeter) in placing brachytherapy needles.
\end{abstract}

\section{Introduction}

In traditional brachytherapy procedures, the needles are inserted transperineally under the guidance of transrectal ultrasound (TRUS) images. Both the needle and the TRUS are operated manually; the seeds are deposited using a manual applicator. The needles are inserted through fixed holes of a physical template. Therefore, flexibility and maneuverability of needle insertion are severely limited. Sometimes it is difficult to avoid pubic arch (especially for patients with larger prostates) because the needles can only be inserted straight through the template's holes. The consistency and efficiency of the treatment procedure are highly dependent on the clinicians. In order to assist clinicians, it is important to develop a motorized semi-automated robotic system for prostate brachytherapy. Robotic system will not only accurately place the needle and deliver seeds but also can assist less experienced or skillful surgeons to perform the procedures with higher consistency and efficiency.

Several researchers have been developing robotic systems for prostate brachytherapy procedures [1]-[7]. The needle placement robot system developed by 
Fichtinger et al. [1] and Stoianovici et al. [2] comprised a 3 degree-s -freedom (DOF) Cartesian bridge over the patient, a 2DOF remote center $\mathrm{f}$ moti $\mathrm{n}$ (RCM), and a $1 \mathrm{DOF}$ needle insertion with a motorized driver using i.. axially loaded friction transmission. A 7DOF passive arm was employed between the Cartesian stage and the rest two modules (RCM and needle inserter) to position and orient the needle. Although the stages had sufficient encoders, the needle driver lacked precise encoding of the depth of needle insertion which was performed by a friction drive. In this system, seeds can only be deposited manually. Wei et al. [3],[4] and Wan [5] et al. have developed a robotic system for prostate brachytherapy where they have used an industrial robot to position and orient a single hole template through which a needle can be inserted manually. A separate motorized module is used for operating the US probe. The needle placement robot system designed by Kronreif et al. [6], [7] consisted of two offset $\mathrm{x}-\mathrm{y}$ stages which allow positioning and orienting the needle over the perineum in 4DOF (two translational and two rotational). The needle can be inserted through the needle guide manually by the clinician.

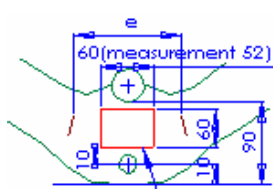

(i)

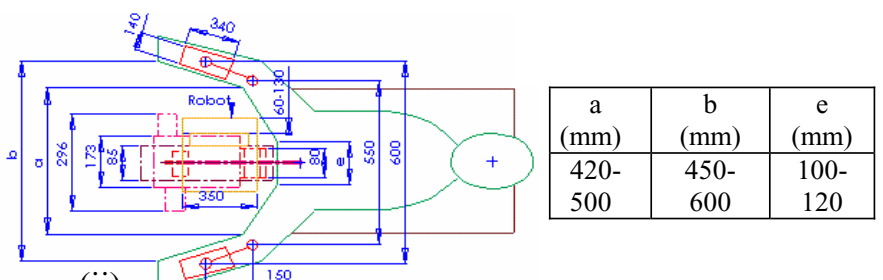

(ii)

Fig. 1. Workspace for robotic-assisted brachytherapy (i) front view, (ii) top view

The available workspace for the robot is quite limited while the patient is in lithotomy position for transperineal prostate brachytherapy (Fig. 1). Thus most of the industrial robots may loose dexterity (or may loose DOF) working in severely constrained workspace in the operating room (OR). Currently, hardly any robotic system is available for full functionalities that are required during actual brachytherapy in the OR. In this paper, we present a robotic system (called EUCLIDIAN - Endo-Uro Computed Lattice for Intratumoral Delivery, Implantation, Ablation with Nanosensing) for brachytherapy that is more equipped to perform most of the required procedures automatically (or semi-automatically). We present a detailed design and development of EUCLIDIAN, sequence of operations in clinical procedures, and experimental results to evaluate EUCLIDIAN's accuracy and repeatability.

\section{System Design and Development}

We have designed and fabricated a more complete robotic system for assisting clinicians during prostate brachytherapy procedures. We collected in-vivo data measuring the available workspace for a robot during actual brachytherapy procedures (Fig. 1); this information helped us in designing a compact robotic system that can work efficiently in the severely constrained workspace in the OR. 


\subsection{Objectives}

The main objects of this robotic system are to (1) Increase accuracy of needle placement and seed delivery, (2) Increase avoidance of critical structures (urethra, pubic arch, bladder), (3) Update dosimetry after each needle is implanted, (4) Detect tissue heterogeneities and deformation via force sensing and imaging feedback, (5) Reduce tediousness and assist clinicians, (6) Reduce trauma and edema, (7) Reduce radiation exposure, (8) Reduce learning curve, and (9) Reduce OR time.

\subsection{Functional Requirements}

The functional requirements of the system are: (1) Quick and easy disengagement in case of emergency, (2) Provision for reverting to conventional manual brachytherapy method at any time, (3) Improvement of prostate immobilization techniques, (4) Provision for periodic quality assurance checking, (5) Updating implant plan after implanting the periphery of the prostate, or most of the needles have been placed and seeds implanted, or any time, (6) A method for the clinician to review and approve the motion plan before the first needle placement, (7) Ability to modulate velocity and needle rotation by automatic feedback control, (8) Visual confirmation by the chosen imaging technique of each seed deposition or the needle tip at the resting position, (9) Steering of the needle by automatic feedback control, (10) Force feedback during needle insertion, and (11) Ease of operation and safety for the patient and OR environment.

\subsection{Prototype Robotic System}

We have designed and fabricated a prototype robotic system for prostate brachytherapy. It comprises of a 9DOF Positioning and a 7DOF Surgery Module.

\subsubsection{Positioning Module}

3 DOF Cart - the cart can move in $\mathrm{x}-\mathrm{y}$ horizontal plane and rotate about a vertical axis. It consisted of a base having four wheels capable of rotating about two of its own axes (a horizontal axis and a vertical axis) and a floor locking mechanism; while locking the cart, it drops four legs and lifts the whole system off the wheels. This ensures rigid locking on any floor and provides greater rigidity to the system. Above the base, all the electronic and electrical components including an industrial computer are housed in an enclosure. The cart is brought near the patient's bed to a relatively rough position and locked on the floor by pressing a lever, then the positioning platform is suitably adjusted.

6DOF Platform - it connects the surgery module to the cart. The platform has 3DOF translational motions and 3DOF rotational motions. Thus it can position and orient the surgery module at any location in the 3D space so that the ultrasound (US) probe can be positioned and oriented in the patient's rectum easily and comfortably, and at the same time the needling mechanism should be suitably aligned with the patient's perineum. The vertical lift (y-motion) of the platform is motorized for ease of operation against gravitational effect. The translational motion in horizontal plane are manual, however these joints are unlocked using two solenoids, for safety and stability they are by default locked. The 3DOF rotational motions (roll-pitch-yaw) are achieved by using a spherical joint which can be mechanically locked at a desired orientation. 


\subsubsection{Surgery Module}

2DOF Ultrasound Probe Driver - the US probe (Acuson ${ }^{\mathrm{TM}} 128 \mathrm{xP}$, Mountain View, CA) can be translated and rotated separately by two DC servo motors fitted with encoders and gear boxes. This enables imaging in transverse plane as well as in sagittal plane, providing capability for improving 3D prostate model for dosimetric planning. Working ranges of motions for US probe are $0-185 \mathrm{~mm}$ and $-91^{\circ}$ to $+91^{\circ}$ in translation and rotation, respectively. The clinician can also drive the US probe manually using the knobs; during this mode the motors are automatically disengaged by the electrical clutches (Fig. 2).The template holder at end of the US probe driver is a provision for manual takeover, if required. The prostate stabilization needle guide can orient the needle at any desired angle in both horizontal and vertical plane resulting in improved stabilization of the prostate and thereby enhanced delivery of the seeds.

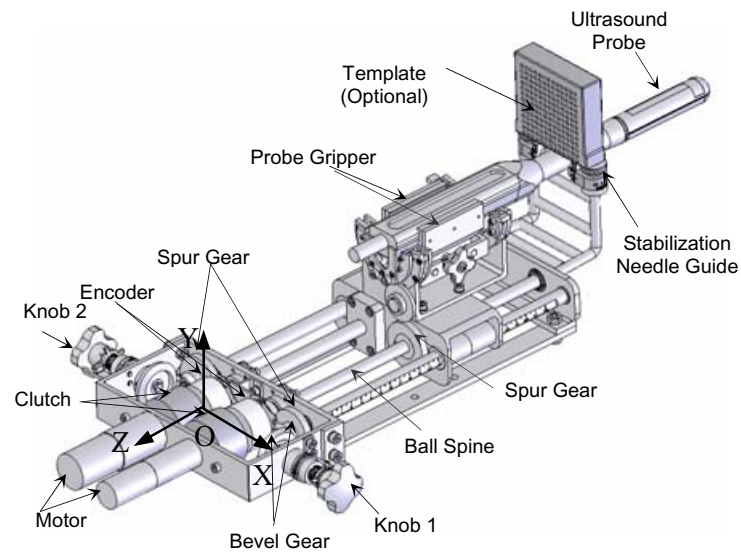

Fig. 2. Ultrasound probe driver

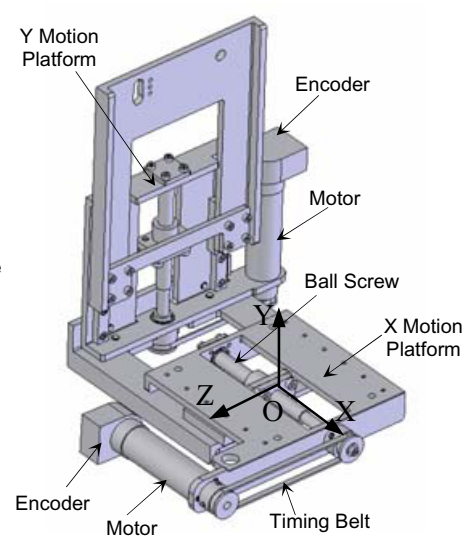

Fig. 3. Gantry robot

3DOF Gantry - it connects the needle driving module to the positioning platform. The gantry has two translational motions ( $\mathrm{x}-\& \mathrm{y}$ - direction) and one rotational motion (pitch). The motions are achieved by DC servo motors and optical encoders fitted with the motors (Fig. 3). The working range of motion of the x-direction is $0-62 \mathrm{~mm}$ and in y-direction is $0-67 \mathrm{~mm}$ which are sufficient to cover the projected treatment area of a conventional template grid $(60 \mathrm{~mm} \times 60 \mathrm{~mm})$. The rotational range for angulating the needle to avoid pubic arch is $-5^{0}$ to $+5^{0}$. The 3DOF motions of the gantry enable to position and orient the needle at any desired location on the patient's perineum with greater freedom due to the absence of a physical template used in conventional brachytherapy. Once the needle is positioned at the desired location close to the perineum, the needle driver inserts the needle into the patient. The motions of the US probe driver and the rest of the surgery module (gantry and needle driver) are decoupled by making two separate open kinematic chains attached to the same positioning platform.

2DOF Needle Driver - the needle which consists of a hollow cannula and a solid stylet are driven separately by two DC servo motors. The cannula is rotated 
continuously or partially using another tiny DC motor. Both the stylet and the cannula are driven from the back (they are pushed); therefore there is no chance of slipping. During actual brachytherapy procedures, the needle traverses through different types of tissues and organs to reach the target in the prostate, and these tissues and organs have different types of boundary conditions that cannot be assessed from experimental data obtained from ex-vivo tissue/organ samples. Therefore, in-vivo measurement of needle insertion force (Figure 4) [9] is very useful in designing and controlling any robotic system that will work in such a constrained space (Fig. 1). In our design process, we have used this in-vivo information. It has been proven that continuous rotation can improve targeting accuracy and can reduce insertion force , whereas partial rotation can increase needle placement accuracy [5],[9].Therefore, we have incorporated the provision for needle rotation in our EUCLIDIAN robot for improving accuracy and seed delivery. To measure and monitor force profiles during the operational procedures, we have installed two single-axis force sensors (Model 13, Honeywell Sensotech, Columbus, $\mathrm{OH}$ ) each at the proximal ends of the stylet and cannula, and one six-axis force-torque sensor (Nano17, ATI Industrial Auto., Apex, $\mathrm{NC}$ ) at the distal end of the cannula (Figure 5). Monitoring of these forces is useful in detecting pubic arch interference (PAI) and will help in assessing needle bending. Unlike commercially available seed cartridges (hold maximum 15 seeds), this cartridge can accommodate 35 seeds at a time resulting in less frequent replacement of the cartridge, and thereby reducing potential radiation exposure as well as reducing OR time.

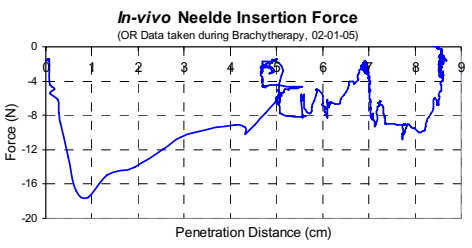

Fig. 4. In-vivo needle insertion force measured during actual prostate brachytherapy procedure (conventional) in the operating room

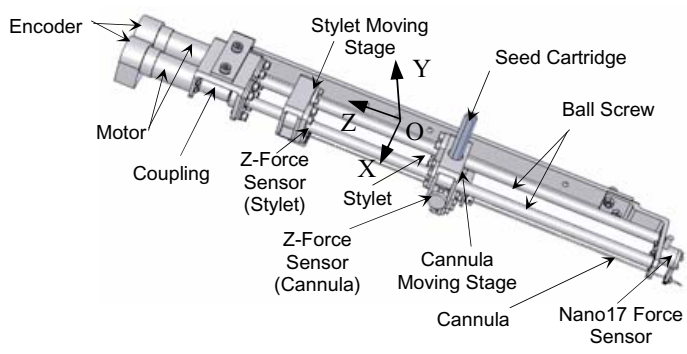

Fig. 5. Needle driver

Seed Pusher - a flat ended style (for beveled-tip brachytherapy needle) is used to push the seed out of the cartridge and to deposit it at the planned locations in the prostate; the stylet's motor is deployed to push the stylet from the proximal end (Fig. 5). All the sequence of motions during the seed delivery is fully automatic; however, at any time the clinician can interrupt the motion. The force sensor at the proximal end of the stylet monitors the force profile on the stylet and thereby confirms the seed removal from the cartridge to the cannula; experiments showed that on an average about $2.5 \mathrm{~N}$ force is required to push the seed out of the cartridge. This is also checked by monitoring the stylet's motor current.

User's Pendant - the handheld pendant with 10 buttons provides the surgeon the freedom to take over the control of the surgery module at any desired time. From the 
user's pendant the surgeon can control the needle insertion, needle rotation, $x-y$ movement of the gantry and system abort.

The EUCLIDIAN system is quite compact to work in the constrained workspace available during prostate brachytherapy procedures (Figs. $6 \& 7$ ). The overall maximum dimensions (length $\mathrm{x}$ width $\mathrm{x}$ height) of the surgery module are $510 \mathrm{~mm} \mathrm{x}$ $290 \mathrm{~mm} \times 235 \mathrm{~mm}$; width at patient's end (distal end) of US Probe Diver is $100 \mathrm{~mm}$ and that of Needle driver is $60 \mathrm{~mm}$. The gross weight of the surgery module is about $9 \mathrm{~kg}$, which can be reduced significantly by using plastic/nylon instead of presently used surgical grade stainless steel and aluminum. All the motors are fitted with optical encoders (MicroMo Electronics, Inc., Faulhaber Group, Clearwater, FL) which provide final motion resolutions (considering gear ratios and screw leads) of $0.0007 \mathrm{~mm}$ for gantry $\mathrm{x}-\mathrm{y}$ translations, $0.004 \mathrm{~mm}$ for stylet and cannula motions; $0.005 \mathrm{~mm}$ and $0.06^{0}$ for US probe translation and rotation, respectively.

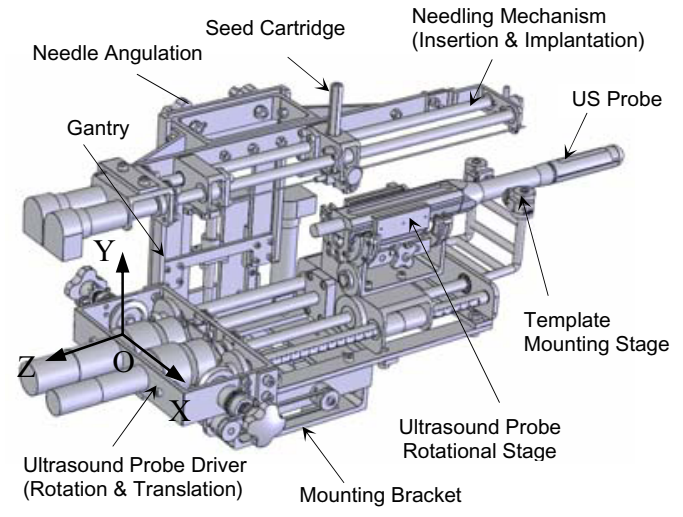

Fig. 6. Assembled surgery module

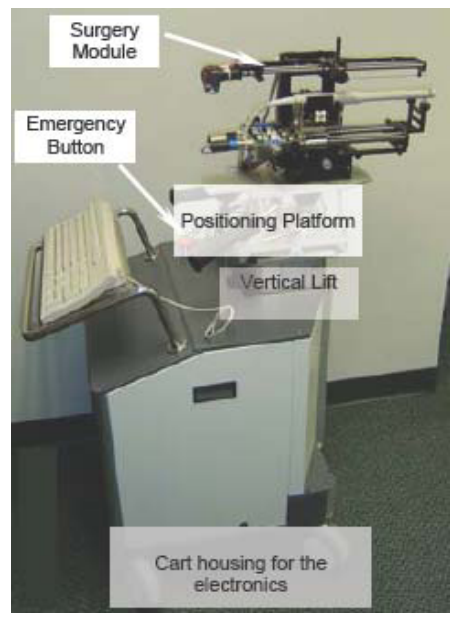

Fig. 7. Prototype robotic system

\section{Clinical Procedures Using the Prototype Robotic System}

Clinical work flow of the prostate brachytherapy procedures using the robotic system is segmented into seven steps as described below.

In setup state, the EUCLIDIAN is initialized and patient information is entered into the computer by the user. Then the TRUS (transrectal ultrasound) is moved to scan the prostate in transversal plane and the images at a desired interval are saved. In the next step, modeling state, the TRUS images are used in delineating the prostate boundary, urethra, pubic bone, rectum, and seminal vesicle. Then a 3D model of the prostate is generated automatically.

Next, this 3D model of the prostate is used for dosimetric planning to obtain the desired coordinates of the radioactive seed distribution. The designed software can display the planned iso-dose contours, needle position and seed locations in 3D. This 
provides the clinicians a useful visualization of the whole treatment plan and if required, the clinicians can edit the plan.

Once the plan is approved by the clinicians, a single needle is inserted into the patient according to the plan. At this stage the TRUS is employed in sagittal plane to tract the needle location. To ensure patient's safety, this needle insertion is performed in a sequential order, i.e. first, the gantry moves in $x-y$ direction to bring the tip of the needle close to the perineum of the patient; second, the gantry is stopped and then the needling mechanism pushed the needle (stylet \& cannula together) into the patient unto a predefined depth; third, the clinician uses the user's pendant to insert the needle up to the final depth. The last stage of user's takeover is to ensure patient's safety as well as to accommodate any change in planned depth that may be required due to tissue/organ deformation or needle deflection. After the needle insertion, the system is in implanting state. Seed is loaded from cartridge and implanted according to the plan, and the needle is withdrawn.

As seeds being delivered, the system can go into a validation state to validate the latest dosimetry, or skip the validation and return to the needling state. In validation state, the prostate is scanned and seeds are located in order to update the dosimetry for validation purpose. If the clinicians find that the dosimetry is not satisfactory, the system can return to the planning state to make adjustment for the remaining seeds to be inserted.

The patient safety is ensured by a variety of hardware (sensors and stops) and software checks. An emergency button on the top of the cart can be used to stop the whole system in case of emergency. The clinician can move the needle and gantry at any desired speed by using the user's pendant. He can also abort the any motion at any time using the pendant. Although the system can execute coordinated (or simultaneous) motion of all the axes, we preferred to have sequential motions of the critical components such as needle insertion, seed delivery, and needle withdrawal for patient's safety reasons. However, to optimize total operating time some of the motions (US probe and gantry) are coordinated. Sterilization of needle passages and seed passages have been ensured by incorporating sterilized inserts. In this prototype system, these inserts are permanent; however, for routine use they can be of

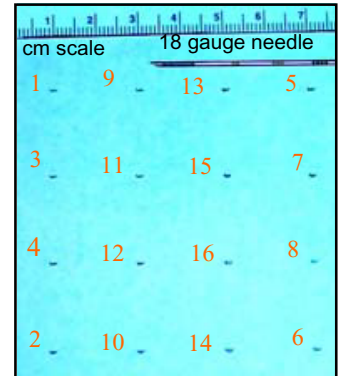

(a)

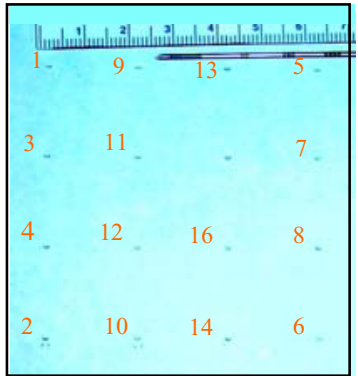

(b)

Fig. 8. Position and size of holes (a) after one hundred penetrations at each location by moving the needle from one location to the next location after each penetration, and (a) after single penetration at each location 
disposable type. Most of the parts of the EUCLIDIAN robot are isolated from patient's blood and fluids by using leather jacket for the positioning module and drapes for the TRUS driver, gantry, and needling mechanism. The cleaning and decontamination of the relevant portions of the system is performed the same way as that currently practiced in the OR.

\section{Results and Discussions}

To evaluate the system's accuracy and repeatability we performed some preliminary experiments. The system was run in a pattern (as shown in Fig. 8) for about 2 hours at a time. An 18 gauge beveled-tip brachytherapy needle was inserted into a graph paper pasted on a block of foam. There were a total of 16 penetration locations in a $60 \mathrm{~mm} x$ $60 \mathrm{~mm}$ area. The gantry moved the needle driver in a sequence (1-2-3- ..-16), as shown in Fig. 8(a)) so that the mechanical systems go through back and forth motion to experience backlash/slack errors in the motion transfer trains/linkages. After a single penetration at each location the needle was moved to the next location, and this was repeated for 100 times. The $\mathrm{x}$ and $\mathrm{y}$ speed of the gantry was $10 \mathrm{~mm} / \mathrm{s}$ and the needle speed was $50 \mathrm{~mm} / \mathrm{s}$. To assess the error and deviation we have also done the same for a single insertion at each location (Fig. 8(b)). It was very difficult to distinguish the deviation of the penetration holes even after 100 runs. We magnified the image of the holes by 5 times to measure the errors. The average errors in $\mathrm{x}$ and $\mathrm{y}$ motions that the system (needle tip) encountered after 100 insertions was $\pm 0.15 \mathrm{~mm}$ with repeatability of $\pm 0.05 \mathrm{~mm}$.

\section{Remarks and Future Work}

This paper presented the design and development of a compact robotic system for prostate brachytherapy treatment with radioactive permanent seed deposition. Numerous techniques and sensors were incorporated into the prototype to improve the needle insertion accuracy, and thereby reducing the seed delivery errors; this ensures improved radiation dose delivery to the patient. The system can be operated in both automated and semi-automated modes. In case of any emergency the system can be stopped instantaneously and the clinicians can switch to conventional manual mode. The patient safety is ensured by a variety of hardware (sensors and stops) and software checks. Accuracy and repeatability of the prototype system were tested and they were found to be very accurate. We are performing more rigorous experiments with phantoms to evaluate the overall performance of the system. In addition to US imaging modality we are considering the use of CT (computed tomography) as more precise imaging modality. Once the phantom studies are completed we intend to start clinical trials.

\section{Acknowledgements}

This work is supported by the National Cancer Institute, under grant R01 CA091763. 


\section{References}

1. Fichtinger, G., DeWeese, T. L., Patriciu, A., et al. System for Robotically Assisted Prostate Biopsy and Therapy with Intraoperative CT Guidance. Acad. Radio., Vol. 9, (2002) 60-74.

2. Stoianovici, D., Cleary, K., Patriciu, A., et al. AcuBot: A Robot for Radiological Percutaneous Interventions. IEEE Trans. on Rob. and Auto., Vol. 19(5), (2003) 927-930.

3. Wei, Z., Wan, G., Gardi, L., et al. Robot-assisted 3D-TRUS Guided Prostate Brachytherapy: System Integration and Validation. Medical Physics, Vol. 31(3), (2004) 539-548.

4. Wei, Z., Wan, G., Gardi, L., Downey, D.B., and Fenster, A.: Robotic Aided 3D TRUS Guided Intraoperative Prostate Brachytherapy. SPIE, Vol. 5367, (2004) 361-370.

5. Wan, G., Wei, Z., Wan, G., Gardi, L., Downey, D.B., and Fenster, A.: Brachytherapy Needle Deflection Evaluation and Correction. Medical Physics, Vol. 32(4), (2005) 902-909.

6. Kettenbach, J., Kronreif, G., Figl, M., et al. Robot-assisted Biopsy using Computed Tomography-guidance: Initial Results from In Vitro Tests. Investigative Radiology, Vol. 40, (2005) 219-228.

7. Kettenbach, J., Kronreif, G., Figl, M., et al. Robot-assisted Biopsy using Ultrasound Guidance: Initial Results from In Vitro Tests. European Radiology, Vol. 15, (2005) 765-71.

8. Podder, T.K., Clark, D.P., Sherman, J., Messing, E.M., Fuller, D., Rubens, D.J., et al. In vivo Motion and Force Measurement of Surgical Needle Intervention during Prostate Brachytherapy. Medical Physics Journal, Vol. 33(8), (2006).

9. Podder, T.K., Liao, L., Sherman, J., Misic, V., Zhang, et al. Assessment of Prostate Brachytherapy and Breast Biopsy Needle Insertions and Methods to Improve Targeting Accuracy. The IFMBE Proc. of the Int. Conf. on Biomed. Eng. (ICBME), Vol. 12, (2005). 